\title{
Pulsational constraints to the metallicity gradient in the Galactic disk
}

\author{
F. Caputo ${ }^{1}$, M. Marconi ${ }^{2}$, I. Musella ${ }^{2}$, and F. Pont ${ }^{3}$ \\ 1 Osservatorio Astronomico di Roma, Via di Frascati 33, 00040 Monteporzio Catone, Italy \\ 2 Osservatorio Astronomico di Capodimonte, Via Moiariello 16, 80131 Napoli, Italy \\ 3 Observatoire de Genève, Switzerland \\ e-mail: caputo@coma.mporzio.astro.it; marcella@na.astro.it; ilaria@na.astro.it; \\ frederic.pont@obs.unige.ch
}

Received 11 December 2000 / Accepted 26 March 2001

\begin{abstract}
BVI data of Galactic Cepheids observed in the solar neighborhood and in the outer disk are analyzed on the basis of nonlinear, non-local and time-dependent convective pulsating models computed for different assumptions on the chemical composition. We show that the theoretical color-color, period-luminosity, and period-luminosity-color relations, as predicted by the models with varying metal content, provide the way to simultaneously estimate the true distance modulus $\mu_{0}$ and the metal content $Z$ of each individual variable. On this basis, we estimate that the metallicity distribution of Cepheids with Galactocentric distances $d$ from $\sim 6$ to $\sim 19 \mathrm{kpc}$ can be represented by a linear gradient of $-0.05 \pm 0.01 \mathrm{dex} \mathrm{kpc}^{-1}$, which is close to previous empirical determinations based on Cepheids, Planetary Nebulae and HII regions. However, our results are not excluding a different scenario, such as a two-zone model with a discontinuity in metallicity near $d=10 \mathrm{kpc}$ (see Twarog et al. 1997).
\end{abstract}

Key words. stars: variables: Cepheids - stars: oscillations - stars: distances

\section{Introduction}

Thanks to the characteristic period-luminosity (PL) relation, classical Cepheids are primary standard candles to set distances within the Local Group, as well as to calibrate secondary distance indicators for more distant galaxies. Consequently, they are fundamental milestones on the path to estimate the value of the Hubble constant $H_{0}$, thus providing valuable clues on the cosmological age problem.

A large uncertainty on the Cepheid distance scale comes from the possible metallicity dependence of both the slope and the zero-point of the PL relation. This is an important point since current distance determinations use empirical multiwavelength PL relations based on the Cepheids in the Large Magellanic Cloud (LMC), and a correction may be necessary if the Cepheids whose distance we are determining have a different metal abundance from the LMC variables $(<[\mathrm{Fe} / \mathrm{H}]>\sim-0.30$, see Luck et al. 1998).

So far the empirical studies have suggested that the PL slope for Galactic and LMC Cepheids is almost identical

Send offprint requests to: M. Marconi,

e-mail: marcella@na.astro.it (see Feast 1999 and references therein), but more recent investigations seem to support the hypothesis that the Galactic PL relation may be shallower than that observed for LMC variables (Groenewegen \& Oudmaijer 2000). As for the theoretical side, almost all the calculations of linear pulsating models (Chiosi et al. 1993; Saio \& Gautschy 1998; Baraffe et al. 1998; Alibert et al. 1999; Sandage et al. 1999) predict rather metal-insensitive PL relations. However, it should be noted that the linear approach does not take in the due account the coupling between pulsation and convection, which is fundamental to get reliable predictions about the red edge of the instability strip. As a consequence, the predicted PL relation is derived by simply placing the pulsation red edge at a fixed temperature shift relative to the blue edge or by assuming an arbitrary efficiency of the convective transport (see discussion in Bono et al. 2000).

On the contrary, recent nonlinear, non-local and timedependent convective pulsating models computed with various chemical abundances show that an increase in the total metal content $Z$ moves the instability strip towards cooler effective temperature, and that consequently the bolometric magnitude of metal-rich pulsators is on average fainter, for fixed period, than that of metal-poor 
ones (Bono et al. 1999a; Bono et al. 1999b). As a result, both the slope and zero-point of the predicted PL relations at the various wavelengths, as well as the predicted period-luminosity-color (PLC) and color-color (CC) relations (Caputo et al. 2000), turn out to depend, to different extent, on the pulsator metallicity. Based on these theoretical relations, it has been already suggested (Caputo et al. 1999) that observations in three bands could provide selfconsistent estimates for the Cepheid distance, reddening and metallicity.

The possibility of deriving simultaneously distance and metallicity for Cepheids with a wide range of Galactic positions is of particular relevance for abundance gradient studies. In this paper we intend to take advantage of the metal-dependent theoretical relations provided by the convective pulsating models to derive information on the metallicity gradient in the Galactic disk. To this purpose, we adopted $B V I$ data of Cepheids observed in the solar neighborhood (Laney \& Stobie 1993, hereinafter LS93) ${ }^{1}$ and towards the Galactic anti-center (Pont et al. 1997, hereinafter P97).

The adopted procedure is outlined in Sect. 2, which also contains a test of its internal reliability. Application to Galactic Cepheids is discussed in Sect. 3, while the resulting metallicity gradient in the Galactic disk is presented in Sect. 4. In Sect. 5 we show the effect on the adopted procedure of replacing the theoretical color-color relations with a set of empirical ones. The concluding remarks close the paper.

\section{The adopted $B V I$ method}

The nonlinear convective pulsating models adopted in this paper are computed with four values of the stellar mass (5, 7,9 , and $\left.11 M_{\odot}\right)$ and three chemical compositions $(Y=$ $0.25, Z=0.004 ; Y=0.25, Z=0.008 ; Y=0.28, Z=$ $0.02)$. The discussion on the input physics and computing procedures have been already presented (see Bono et al. 1999a; Bono et al. 1999b) and will not be repeated. The models provide the pulsation amplitude and the average magnitudes of the pulsator. Consequently the predicted $\mathrm{PL}, \mathrm{PLC}$ and $\mathrm{CC}$ relations are derived in the BVRIJK bands (see also Caputo et al. 2000). Note that, since our pulsating models have masses $M \geq 5 M_{\odot}$, the relations presented in this paper hold for fundamental pulsators with period larger than $\sim 3$ days (see Bono et al. 1999, 2000).

Table 1 gives the CC and PLC relations used in the present paper, as obtained from fundamental pulsating models with the labeled metal content. Since empirical studies adopt different methods of averaging observed magnitudes and colors over the full pulsation cycle, the

\footnotetext{
1 To the intensity mean $B V$ magnitudes we added the $I$ magnitudes as derived from $\langle V\rangle-\langle I\rangle-(V-I)=$ $-0.03 \mathrm{mag}$, where the magnitude-averaged $(V-I)$ colors are by Caldwell \& Coulson (1987). For details see Caputo et al. (2000).
}

Table 1. Theoretical relations for magnitude averaged and intensity averaged quantities, as a function of metal content.

\begin{tabular}{|c|c|c|c|c|}
\hline$\overline{\mathrm{Z}}$ & $\bar{a}$ & $\bar{b}$ & $\overline{c c}$ & $\overline{\sigma \sigma}$ \\
\hline \multicolumn{5}{|c|}{$(B-V)=a+b(V-I)$} \\
\hline 0.004 & $-0.35 \pm 0.01$ & $+1.32 \pm 0.02$ & & 0.01 \\
\hline 0.008 & $-0.29 \pm 0.01$ & $+1.29 \pm 0.03$ & & 0.01 \\
\hline 0.02 & $-0.18 \pm 0.01$ & $+1.23 \pm 0.02$ & & 0.01 \\
\hline \multicolumn{5}{|c|}{$<B>-<V>=a+b[<V>-<I>]$} \\
\hline 0.004 & $-0.33 \pm 0.01$ & $+1.28 \pm 0.02$ & & 0.01 \\
\hline 0.008 & $-0.30 \pm 0.01$ & $+1.28 \pm 0.03$ & & 0.01 \\
\hline 0.02 & $-0.24 \pm 0.01$ & $+1.28 \pm 0.02$ & & 0.01 \\
\hline \multicolumn{5}{|c|}{$\left(M_{I}\right)_{\text {blue }}=a+b \log P+c \log P^{2}$} \\
\hline 0.004 & $-1.24 \pm 0.01$ & $-4.86 \pm 0.06$ & $+0.76 \pm 0.01$ & 0.03 \\
\hline 0.008 & $-1.33 \pm 0.01$ & $-4.69 \pm 0.01$ & $+0.77 \pm 0.02$ & 0.03 \\
\hline 0.02 & $-1.99 \pm 0.01$ & $-3.09 \pm 0.01$ & $+0.16 \pm 0.01$ & 0.03 \\
\hline
\end{tabular}

\begin{tabular}{ccccc}
\multicolumn{5}{c}{$\left(M_{I}\right)_{\text {red }}=a+b \log P+c \log P^{2}$} \\
0.004 & $-1.55 \pm 0.01$ & $-3.74 \pm 0.01$ & $+0.45 \pm 0.01$ & 0.03 \\
0.008 & $-1.63 \pm 0.01$ & $-3.45 \pm 0.03$ & $+0.36 \pm 0.02$ & 0.03 \\
0.02 & $-2.00 \pm 0.01$ & $-2.70 \pm 0.02$ & $+0.17 \pm 0.01$ & 0.03 \\
\hline \multicolumn{5}{c}{} \\
& $<M_{I}>_{\text {blue }}=a+b \log P+c \log P^{2}$ \\
0.004 & $-1.29 \pm 0.01$ & $-4.80 \pm 0.06$ & $+0.73 \pm 0.01$ & 0.03 \\
0.008 & $-1.38 \pm 0.01$ & $-4.60 \pm 0.01$ & $+0.72 \pm 0.01$ & 0.03 \\
0.02 & $-1.98 \pm 0.01$ & $-3.16 \pm 0.01$ & $+0.19 \pm 0.01$ & 0.03 \\
\hline \multicolumn{5}{c}{} \\
0.004 & $-1.55 \pm 0.01$ & $-3.75 \pm 0.01$ & $+0.45 \pm 0.01$ & 0.03 \\
0.008 & $-1.61 \pm 0.01$ & $-3.49 \pm 0.02$ & $+0.38 \pm 0.01$ & 0.03 \\
0.02 & $-2.00 \pm 0.01$ & $-2.71 \pm 0.03$ & $+0.17 \pm 0.01$ & 0.03 \\
\hline
\end{tabular}

\begin{tabular}{ccccc}
\multicolumn{5}{c}{$\left(M_{I}\right)=a+b \log P+c(V-I)$} \\
0.004 & $-3.62 \pm 0.04$ & $-3.66 \pm 0.03$ & $+2.90 \pm 0.09$ & 0.03 \\
0.008 & $-3.64 \pm 0.04$ & $-3.67 \pm 0.04$ & $+2.92 \pm 0.09$ & 0.03 \\
0.02 & $-3.72 \pm 0.05$ & $-3.62 \pm 0.06$ & $+2.96 \pm 0.13$ & 0.03 \\
\hline \multicolumn{5}{c}{} \\
$5 M_{I}>=a+b \log P+c[<V>-<I>]$ \\
0.004 & $-3.55 \pm 0.03$ & $-3.58 \pm 0.03$ & $+2.75 \pm 0.07$ & 0.03 \\
0.008 & $-3.54 \pm 0.03$ & $-3.59 \pm 0.02$ & $+2.74 \pm 0.06$ & 0.03 \\
0.02 & $-3.61 \pm 0.03$ & $-3.59 \pm 0.04$ & $+2.85 \pm 0.09$ & 0.03 \\
\hline
\end{tabular}

relations are given for both magnitude-averaged $\left[\left(M_{i}\right)\right.$, $\left.\left(M_{i}-M_{j}\right)\right]$ and intensity-averaged $\left[\left\langle M_{i}\right\rangle,\left\langle M_{i}\right\rangle-\right.$ $<M_{j}>$ ] quantities. As for the PL relation, it is well known that it is a "statistical" solution which depends on the edges of the pulsation region as well as on the distribution of pulsators within the instability strip (see Caputo et al. 2000). In other words, the PL relation of Cepheids with the same distance, reddening and metallicity has a 
"tolerance" which is due to the finite width of the pulsation region. However, our pulsating models provide a firm knowledge of the edges of the instability strip, and for this reason we list in Table 1 the PL relations in the $I$-band at the blue and the red limit for fundamental pulsation. These are quadratic relations due to the nonlinearity of the PL correlation in the optical bands (see also Caputo et al. 2000). It seems worth noticing that, under the above assumptions that all the variables have the same distance, reddening and metallicity, the intrinsic scatter of the PLC relation is quite negligible. On very general grounds, the pulsation period is correlated with the mass, luminosity and effective temperature of the pulsator. Moreover, stellar evolution theory gives the massluminosity relation for each given chemical composition. It follows that, once metallicity and reddening are known, the luminosity can be accurately described in terms of period and color.

As a first step of our procedure, the predicted $B-V$ versus $V-I$ relations are used to derive the individual reddening $E(B-V)$ at each adopted metallicity. As for the ratio $A_{V} / E(B-V)$ and $A_{I} / E(B-V)$ we follow the relations

$$
A_{V}=\left(3.07+0.28(B-V)_{0}+0.04 E(B-V)\right) E(B-V)
$$

$$
A_{I}=\left(1.82+0.205(B-V)_{0}+0.022 E(B-V)\right) E(B-V)(2)
$$

from Dean et al. (1978, hereafter DWC) and LS93.

By using the $B V I$ reddening and the corresponding $\mathrm{PLC}_{V I}$ and $\mathrm{PL}_{I}$ relations, we derive at each given metallicity three estimates for the Cepheid true distance modulus, namely $\mu_{0}\left(\mathrm{PLC}_{V I}\right), \mu_{0, \text { red }}\left(\mathrm{PL}_{I}\right)$, and $\mu_{0 \text {,blue }}\left(\mathrm{PL}_{I}\right)$. As already stated by Caldwell \& Coulson (1986), PL and PLC distances show an opposite behavior at varying metallicity. As a fact, we show in Fig. 1 that the value of $\mu_{0}\left(\mathrm{PLC}_{I}\right)$ increases with increasing the adopted $Z$ (filled circles), whereas both the values for $\mu_{0}\left(\mathrm{PL}_{I}\right)$ tend to decrease (open circles and squares). On this basis, we are in condition to derive the two estimates $\log Z_{\text {blue }}$ and $\log Z_{\text {red }}$ at which the identity $\mu_{0}\left(\mathrm{PL}_{I}\right)=\mu_{0}\left(\mathrm{PLC}_{V I}\right)$ is satisfied. Then, the arithmetic mean $\left(\log Z_{\text {blue }}+\log Z_{\text {red }}\right) / 2$ and the semi-difference $\left(\log Z_{\text {blue }}-\log Z_{\text {red }}\right) / 2$ are taken as the average metallicity of the variable and its internal error, respectively. At the same time, from the two values $\mu_{0 \text {,blue }}$ and $\mu_{0, \text { red }}$ we obtain the Cepheid average true distance modulus and its internal error.

In order to test the internal accuracy of the adopted relations, we considered the fundamental pulsating models with $Z=0.004,0.008$, and 0.02 at the same distance modulus $\mu_{0}=10.50 \mathrm{mag}$. Following the above procedure, we eventually get that the average metallicity $(\langle Z\rangle=$ $0.004 \pm 0.002,0.008 \pm 0.002$ and $0.020 \pm 0.005)$ and true distance modulus $\left(<\mu_{0}\right\rangle=10.48 \pm 0.05 \mathrm{mag}, 10.49 \pm$ $0.05 \mathrm{mag}$ and $10.42 \pm 0.06 \mathrm{mag})$ of the three samples are in close agreement with the input values.

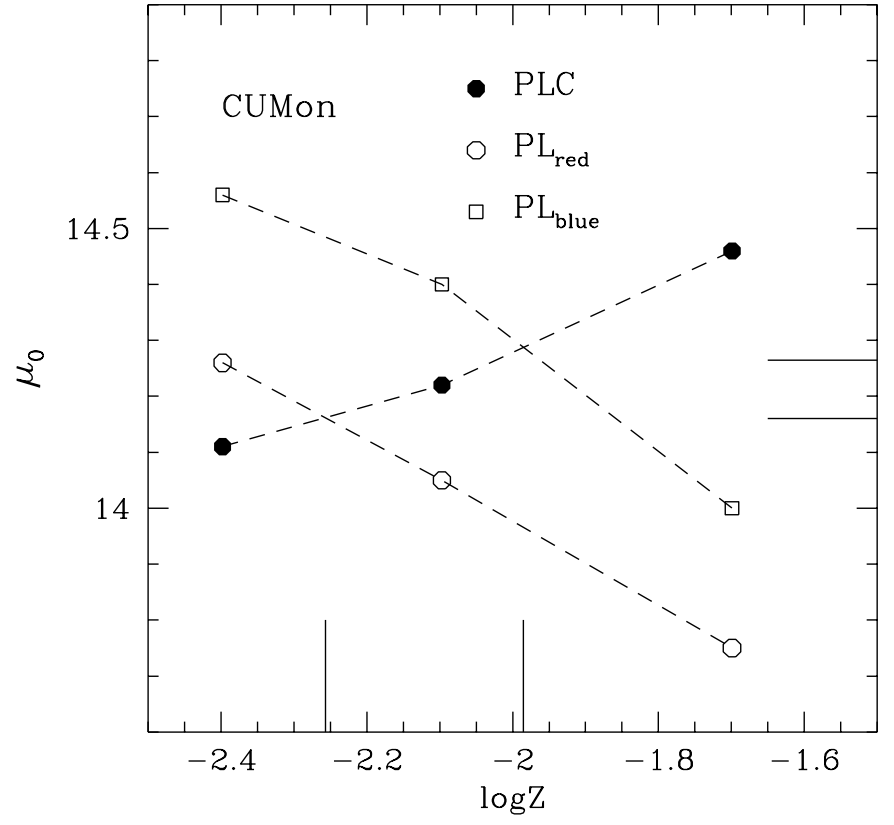

Fig. 1. Dependence of $\mu_{0}\left(\mathrm{PL}_{I}\right)$ and $\mu_{0}\left(\mathrm{PLC}_{V I}\right)$ on the adopted metallicity (dashed lines) for the Cepheid CU Mon. The vertical solid lines depict the maximum and minimum metallicity, while the horizontal ones depict the maximum and minimum true distance modulus (see text).

\section{Application to observed Cepheids}

Figure 2 shows the Cepheids observed by LS93 (lower panel) and P97 (upper panel) in comparison with the intrinsic $\mathrm{CC}$ relations in Table 1 for intensity-averaged and magnitude-averaged colors, respectively. The dasheddotted line shows the reddening vector with $E(V-$ $I) / E(B-V) \sim 1.3$ (see Eqs. (1) and (2)).

In order to show how the adopted procedure is working, we plot in Fig. 3 the absolute $I$-magnitudes of LS93 Cepheids, as inferred from $\mu_{0}\left(\mathrm{PLC}_{V I}\right)$, in comparison with the predicted edges of the instability strip (solid lines) listed in Table 1.

The figure clearly shows that almost all the Cepheids in the solar neighborhood have a metallicity larger than $Z=0.004$ (as a fact, they are fainter than the red edge of the instability strip with $Z=0.004$ ), and very few ones have a metallicity lower than $Z=0.02$ (only those which are brighter than the blue edge of the instability strip with $Z=0.02)$. As for the outer disk Cepheids plotted in Fig. 4, we find just a handful with metallicity larger than $Z=0.008$ (only those which are fainter than the red edge of the instability strip with $Z=0.008$ ). More in details, the plot of derived $Z$ versus period for individual Cepheids presented in Fig. 5 shows that the average metallicity of nearby and distant Cepheids is $\langle\log Z\rangle=-1.93 \pm 0.23$ and $\langle\log Z\rangle=-2.24 \pm 0.23$, respectively.

Before proceeding, it seems worth comparing the "pulsational" metallicities with empirical spectroscopic $[\mathrm{Fe} / \mathrm{H}]$ measurements. Figure 6 shows that our estimates for the total metallicity $Z$ are in fair agreement with available $[\mathrm{Fe} / \mathrm{H}]$ data for Cepheids in the Galaxy and Magellanic 


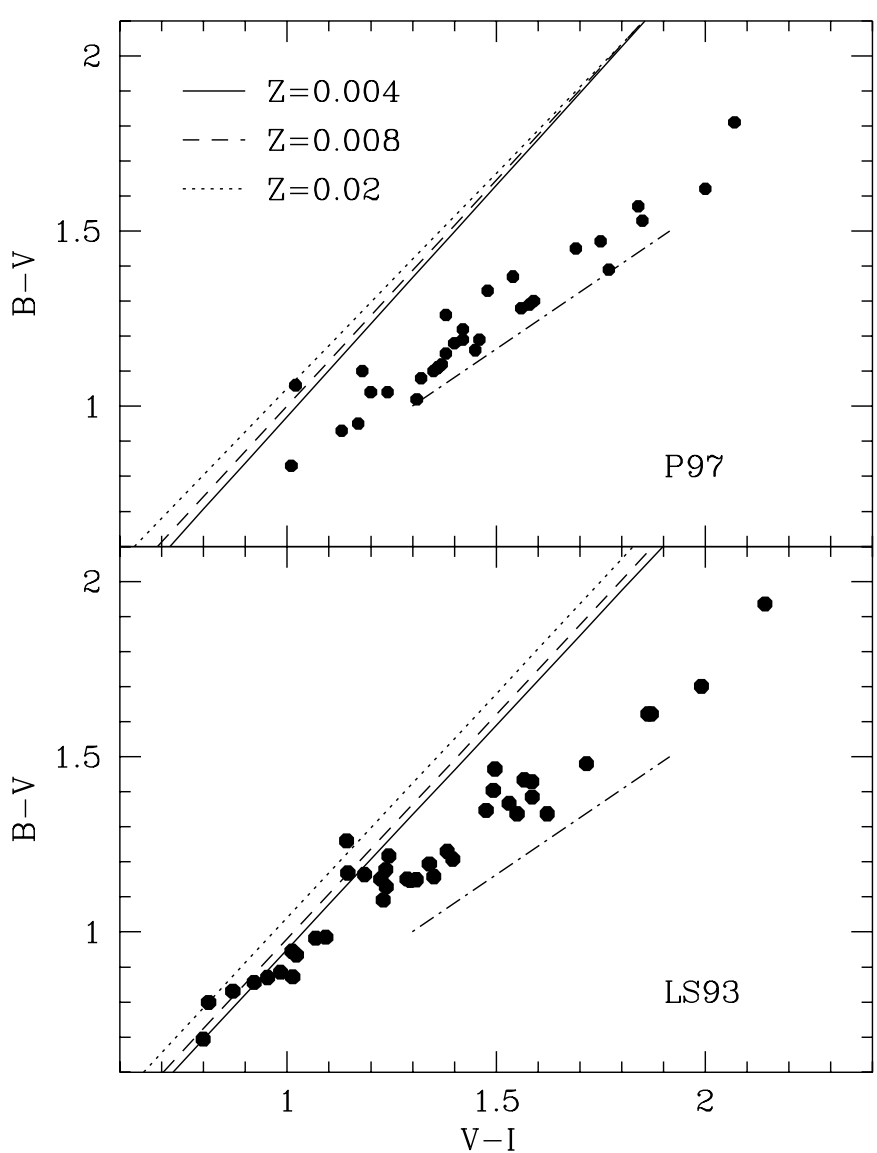

Fig. 2. Intensity-averaged colors by LS93 (lower panel) and magnitude-averaged colors by P97 (upper panel) in comparison with the intrinsic CC relations in Table 1. The dashed-dotted line depicts the reddening vector.

Clouds (see Fry \& Carney 1997; Luck et al. 1998, respectively). With the only exception of the LMC variable HV909 (not plotted in the figure), the Cepheids follow on average the canonical relation $\log Z \sim[\mathrm{Fe} / \mathrm{H}]-1.7$ (dashed line). Specifically, for the Galactic Cepheids $(<[\mathrm{Fe} / \mathrm{H}]>=$ $-0.05 \pm 0.10)$ we get $\langle\log Z\rangle=-1.89 \pm 0.20$, while for $\operatorname{LMC}(<[\mathrm{Fe} / \mathrm{H}]>=-0.30 \pm 0.13)$ and $\operatorname{SMC}(<[\mathrm{Fe} / \mathrm{H}]>=$ $-0.75 \pm 0.07)$ variables we obtain $\langle\log Z>=-2.05 \pm 0.20$ and $\langle\log Z\rangle=-2.39 \pm 0.20$, respectively, confirming that our procedure is fully capable to bring out a metallicity variation within a sample of Cepheids. Unfortunately, we have no possibility to test the predicted distance moduli on stars with available measured distances, e.g. Hipparcos data. The Hipparcos distances are too uncertain for any application on individual variables, by several tens of percent even for the brightest Cepheids. In this case the metallicity effect would be totally dominated by this uncertainty, as well as by the tricky biases involved in the treatment of high-relative-error parallax data.

\section{The Cepheid metallicity gradient in the Galactic disk}

Having derived both $Z$ and $\mu_{0}$ for each Cepheid, we are in the position of investigating the correlation between



Fig. 3. Absolute $I$-magnitudes of nearby Cepheids, as inferred from $\mu_{0}\left(\mathrm{PLC}_{V I}\right)$, in comparison with the predicted boundaries for fundamental pulsation.

metallicity and distance from the Galactic center by using published galactic coordinates (Fernie et al. 1995; P97) and by adopting a distance between the sun and the Galactic center of $8.5 \mathrm{kpc}$. The results are presented in Fig. 7, where open and filled circles are solar neighborhood and outer disk Cepheids, respectively, and the dashed line depicts the metallicity gradient of $-0.07 \mathrm{dex} \mathrm{kpc}^{-1}$ earlier suggested by Harris (1981).

As for our results, we start with the traditional method of simply fitting a line to all the data in Fig. 7. From the least squares fit we derive

$\log Z=-0.05( \pm 0.01) d-1.52( \pm 0.10)$

where $d$ is the Cepheid galactocentric distance in kpc. This solution, which is drawn as solid line in the lower panel of Fig. 7, appears in fair agreement with Harris (1981), as well as with recent studies based on Planetary Nebulae (Maciel \& Quireza 1999), HII regions (Afflerbach et al. 1997) and early B-type main sequence stars situated in Galactic open clusters (Rolleston et al. 2000).

However, it is evident from the data in Fig. 7 that a change in metallicity may occur near $10 \mathrm{kpc}$. By breaking 




Fig. 4. As in Fig. 3, but for outer disk Cepheids.

the Cepheid distances into two groups within or beyond $10 \mathrm{kpc}$, we derive

$\log Z=-0.01( \pm 0.05) d-1.78( \pm 0.36)$

for the inner group, and

$\log Z=-0.02( \pm 0.02) d-1.98( \pm 0.23)$

for the outer group. These solutions, which are drawn as solid lines in the upper panel of Fig. 7, yield that the gradients for the two groups of stars are not significantly different from zero, supporting the hypothesis of a two-zone model suggested by Twarog et al. (1997). Unfortunately, there are clearly few stars at large Galactocentric distance and discussion of the "best" statistical model to the data in Fig. 7 is out the reach of the present paper.

We also examined in a preliminary way data in the $K$ band (Caputo et al. in preparation) and found consistency with the distances and reddenings based on the $B V I$ method. This means that the reddening scale is essentially correct.

\section{Empirical color-color relations}

In the previous section, we have adopted the theoretical CC relations to determine the individual reddening of Galactic Cepheids at each adopted metal content. Here we

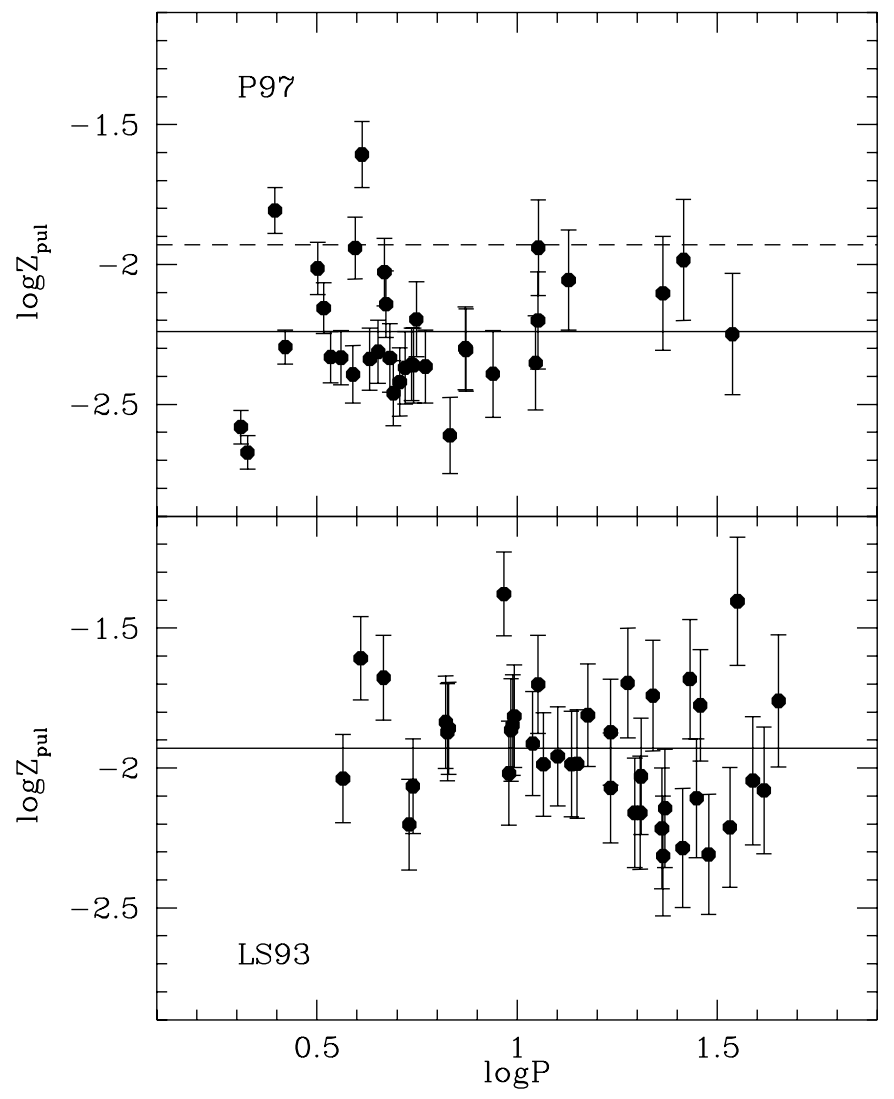

Fig. 5. Individual "pulsational" metallicity as a function of period for P97 (upper panel) and LS93 (lower panel) Cepheid samples. The error bars depict the associated error on the derived metallicity. The solid lines show the average metal content of each sample. The dashed line in the top panel refers to the average metallicity we found for the nearby Cepheids.



Fig. 6. The "pulsational" metallicity $Z_{\text {pul }}$ in comparison with spectroscopic $[\mathrm{Fe} / \mathrm{H}]$ data for Cepheids in the Galaxy and Magellanic Clouds. The dashed line depicts the relation $\log Z=[\mathrm{Fe} / \mathrm{H}]-1.7$. 


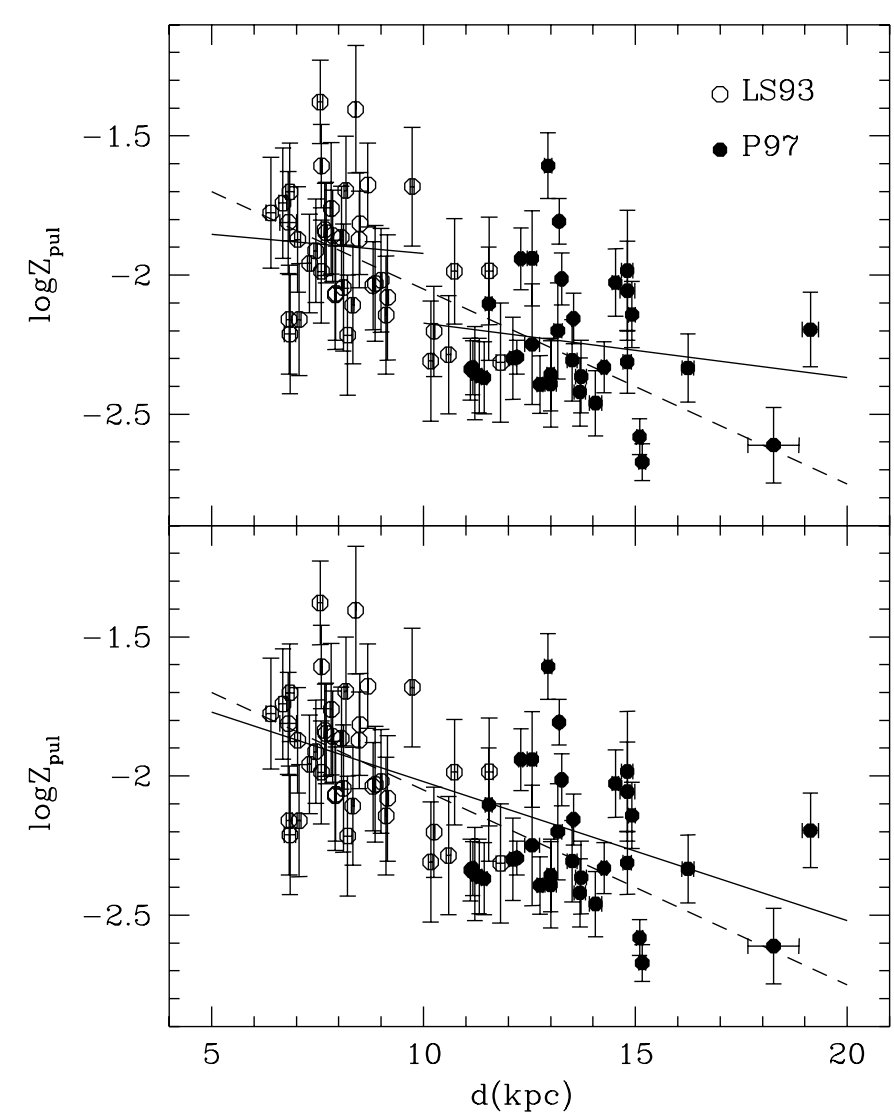

Fig. 7. The Cepheid metallicity gradient in the Galactic disk, as suggested by solar neighborhood (LS93: open circles) and outer disk variables (P97: filled circles). The dashed line depicts

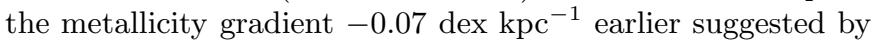
Harris (1981). The solid line in the lower panel shows our linear best fit to all the data (slope $-0.05( \pm 0.01)$ dex $\mathrm{kpc}^{-1}$ ) while those in the upper panel show the solution within $10 \mathrm{kpc}$ (slope $-0.01( \pm 0.05)$ dex $\mathrm{kpc}^{-1}$ ) and beyond $10 \mathrm{kpc}$ (slope $-0.02( \pm 0.02)$ dex $\left.\mathrm{kpc}^{-1}\right)$.

Table 2. Empirical CC relations by Dean et al. (1978) for "normal" Cepheids: $(B-V)_{0}=a+b(V-I)_{0}+c(V-I)_{0}^{2}$.

\begin{tabular}{cccc}
\hline \hline$(B-V)_{0}$ & $a$ & $b$ & $c$ \\
\hline$<0.418$ & -0.063 & 0.78 & 0.425 \\
$\geq 0.418$ & 0 & 0.58 & 0.57 \\
\hline
\end{tabular}

briefly investigate the effects of replacing the above linear relations with the empirical ones derived by DWC from magnitude means of simultaneous $(B-V)$ and $(V-I)$ pairs for Galactic Cepheids with well-known reddening (see Table 2), and extending redwards to $(V-I)_{0} \sim$ $1.1 \mathrm{mag}$. This empirical relation holds for "normal" (i.e. solar metallicity) variables and can be corrected according to the results by Caldwell \& Coulson (1985) in order to account for the two adopted metal abundances $Z=0.008$ and $Z=0.004$. As evident from Fig. 8, the predicted CC relation with $Z=0.02$ agrees with the DWC result with $(V-I)_{0} \leq 0.9 \mathrm{mag}$, while for redder colors it would lead to slightly smaller reddening than the empirical ones. As a fact, we show in Fig. 9 that the difference

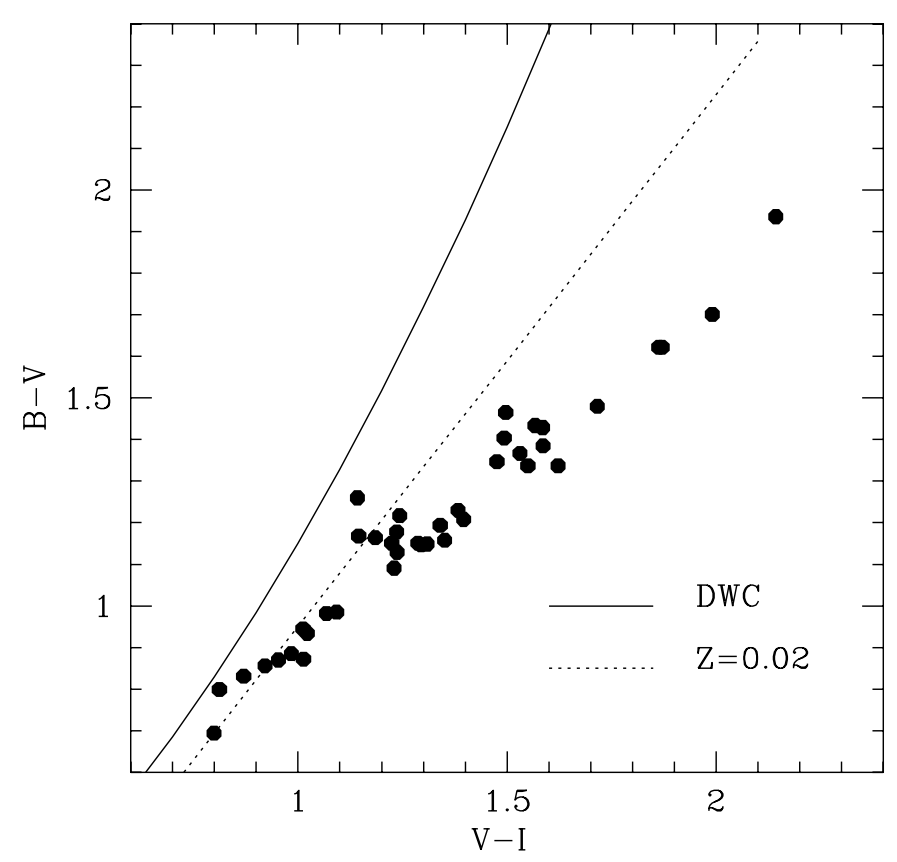

Fig. 8. Intensity-averaged colors by LS93 in comparison with the intrinsic CC relation with $Z=0.02$ (dotted line) and the empirical one in Table 2 (solid line).



Fig. 9. Difference between empirical and predicted reddening with $Z=0.02$ as a function of period for nearby Cepheids.

$\Delta E(B-V)$ between DWC and theoretical results with $Z=0.02$ increases towards the longer periods, i.e. redder colors. As a consequence, if the "empirical" reddenings are used along our procedure to get $Z$ and $\mu_{0}$, then slightly lower metal abundances are derived (see Fig. 10), with the least squares fit through the data giving

$\log Z=-0.05( \pm 0.01) d-1.73( \pm 0.11)$. 


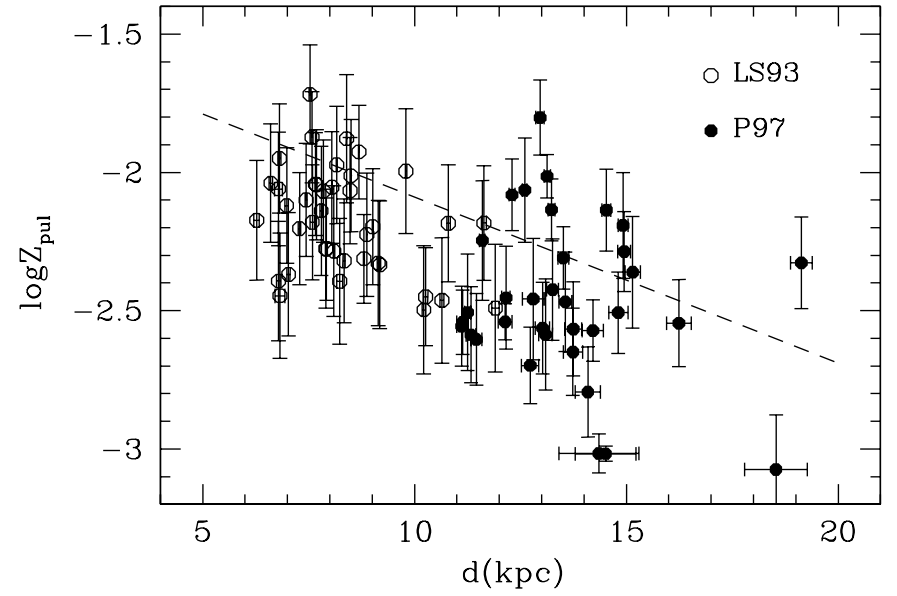

Fig. 10. As in Fig. 7, but with $B V I$ reddening from DWC relation.

\section{Conclusions}

Based upon theoretical convective models of fundamental pulsators with three selected metallicities $(Z=0.004$, 0.008 , and 0.02 ), we present a method to estimate simultaneously the metallicity, reddening and distance of individual Cepheids from $B V I$ data.

The procedure can be summarized as follows:

i) for each adopted metal content $Z$, the predicted $B V I$ Color-Color relation is used to derive the Cepheid reddening. Then, three estimates of the true distance modulus $\mu_{0}$ are obtained from the predicted $\mathrm{PLC}_{V I}$ and $\mathrm{PL}_{I}$ relations (the latter one at both the red and blue edge of the pulsation region);

ii) since $\mu_{0}\left(\mathrm{PLC}_{V I}\right)$ increases whereas $\mu_{0}\left(\mathrm{PL}_{I}\right)$ decreases with increasing $Z$ (see Fig. 1), we derive self-consistent estimates of the Cepheid distance and metallicity (with the associated errors), by demanding the condition $\mu_{0, \mathrm{PL}}=\mu_{0, \mathrm{PLC}}$ (i.e. by requiring that the location of the Cepheid within the instability strip is constrained by the predicted blue and red boundaries of pulsation);

iii) applying such a procedure to Galactic Cepheids, we derive a metallicity gradient in the Galactic disk of $\delta \log Z / \delta d=-0.05( \pm 0.01)$ dex $\mathrm{kpc}^{-1}$, in close agreement with previous results based on Cepheids (Harris 1981), Planetary Nebulae (Maciel \& Quireza 1999), HII regions (Afflerbach et al. 1997) and early B-type stars (Rolleston et al. 2000). However, our results are not excluding a two-zone model with a metallicity discontinuity near $10 \mathrm{kpc}$ (see Twarog et al. 1997). The analysis of more observations of Cepheids at large Galactocentric distance could shed light on the extent and magnitude of the abundance gradient, with useful constraints to the models of disk evolution.

Acknowledgements. It is a pleasure to thank D. Laney for several comments on a preliminary draft of this paper. We are indebted to the referee for valuable suggestions which improved the first version of the article. Financial support for this work was provided by MURST under the scientific project "Stellar observables of cosmological relevance" (Vittorio Castellani, coordinator).

\section{References}

Afflerbach, A., Churchwell, E., \& Werner, M. W. 1997, ApJ, 478,190

Alibert, Y., Baraffe, I., Hauschildt, P., \& Allard, F. 1999, A\&A, 344,551

Baraffe, I., Alibert, Y., M'era, D., Chabrier, G., \& Beaulieu, J. P. 1998, ApJ, 499, 205

Bono, G., Marconi, M., \& Stellingwerf, R. F. 1999a, ApJS, 122, 167

Bono, G., Caputo, F., Castellani, V., \& Marconi, M. 1999b, ApJ, 512, 711

Bono, G., Castellani, V., \& Marconi, M. 2000, ApJ, 529, 293

Caldwell, J. A. R., \& Coulson, I. M. 1985, MNRAS, 212, 879

Caldwell, J. A. R., \& Coulson, I. M. 1986, MNRAS, 218, 223

Caldwell, J. A. R., \& Coulson, I. M. 1987, AJ, 93, 1090

Caputo, F., Marconi, M., \& Musella, I. 2000, A\&A, 354, 610

Caputo, F., Marconi, M., \& Ripepi, V. 1999, ApJ, 525, 784

Chiosi, C., Wood, P. R., \& Capitanio, N. 1993, ApJS, 86, 541

Dean, J. F., Warren, P. R., \& Cousins, A. W. 1978, MNRAS, 183,569

Feast, M. W. 1999, PASP, 111, 775

Fernie, J. D., Beattie, B., Evans, N. R., \& Seager, S. 1995, IBVS, No. 4148

Fry, A. M., \& Carney, B. W. 1997, ApJ, 113, 1073

Groenewegen, M. A. T., \& Oudmaijer, R. D. 2000, A\&A, 356, 849

Harris, H. C. 1981, AJ, 86, 707

Laney, C. D., \& Stobie, R. S. 1993, MNRAS, 263, 291

Luck, R. E., Moffett, T. J., Barnes, T. G., \& Gieren, W. P. 1998, ApJ, 115, 605

Maciel, W. J., \& Quireza, C. 1999, A\&A, 345, 629

Pont, F., Queloz, D., Bratschi, P., \& Mayor, M. 1997, A\&A, 318,416

Rolleston, W. R. J., Smart, S. J., Dufton, P. L., \& Ryans, R. S. I. 2000, A\&A, 363, 537

Saio, H., \& Gautschy, A. 1998, ApJ, 498, 360

Sandage, A., Bell, R. A., \& Tripicco, M. J. 1999, ApJ, 522, 250

Twarog, B. A., Ashman, K. M., \& Anthony-Twarog, B. J. 1997, AJ, 114, 2556 